\title{
Protracted, Intermittent Outbreak of Salmonella Mbandaka Linked to a Restaurant — Michigan, 2008-2019
}

\begin{abstract}
William D. Nettleton, $\mathrm{MD}^{1,2}$; Bethany Reimink, $\mathrm{MPH}^{3}$; Katherine D. Arends, $\mathrm{MPH}^{3}$; Douglas Potter, MBA ${ }^{1}$; Justin J. Henderson, MPH ${ }^{3}$;
\end{abstract} Stephen Dietrich, $\mathrm{MS}^{3}$; Mary Franks, $\mathrm{MPH}^{1}$

In 2018, Michigan public health officials determined that a single restaurant in southwest Michigan was the source for a protracted, intermittent outbreak of Salmonella enterica serotype Mbandaka infections occurring since 2008. Isolates from 36 infected persons shared two highly related pulsed-field gel electrophoresis (PFGE) patterns and highly related whole genome sequencing (WGS) subtypes. The initial focus of the local public health investigation on food items rather than food sources (i.e., restaurants) through a questionnaire, difficulty in food history recollection among ill persons, and sporadic case identification over periods from months to years contributed to delayed source identification. The Kalamazoo County Health and Community Services Department (KHCSD) and the Michigan Department of Health and Human Services (MDHHS) collected clinical specimens, performed multiple rounds of environmental testing, and conducted multiple regulatory visits, and based on accumulated findings over 10 years, identified the restaurant source. A 2018 investigation by KCHCSD and MDHHS found that environmental samples and stool specimens from asymptomatic restaurant employees tested positive for the Salmonella Mbandaka outbreak strain. A complex association between the restaurant environment and employees resulted in patron illnesses. Environmental health interventions, facility renovation, asymptomatic employee exclusions, employee health monitoring, and recurrent facility environmental sampling measures were implemented. As a result of ongoing cases and environmental persistence of Salmonella Mbandaka, the restaurant closed permanently in 2018. Restaurant employee stool testing and environmental sampling for Salmonella early during the investigation of confirmed Salmonella cases linked to a restaurant enhances source identification. Exclusion or restriction of asymptomatic food workers with stool-positive nontyphoidal Salmonella should be considered part of restaurant outbreak mitigation.

\section{Epidemiologic Investigation}

In 2012, KCHCSD was notified by MDHHS about Salmonella Mbandaka cases occurring intermittently since 2008 that were highly related by PFGE pattern. During 2012-2014, a restaurant was not yet associated, so a hypothesis-generating questionnaire was used to ensure capture of detailed patient food histories, which included closed-ended questions about frequently eaten food items, types of restaurants visited, and animal contact. In 2014, although investigations into common suppliers among several restaurants mentioned in food histories were ongoing and other restaurants were named by cases, as more information was collected from supplementary questionnaires, KCHCSD, MDHHS, and the Michigan Department of Agriculture and Rural Development discussed the association of a single restaurant (restaurant A) based on five known, confirmed cases to date reporting a meal at restaurant A. KCHCSD gathered additional information from restaurant $\mathrm{A}$

\section{INSIDE \\ 1114 Disparities in Excess Mortality Associated with COVID-19 - United States, 2020 \\ 1120 Use of Rapid Antigen Testing for SARS-CoV-2 in Remote Communities - Yukon-Kuskokwim Delta, Alaska, September 15, 2020-March 1, 2021 \\ 1124 Notes from the Field: Vitamin D-Deficient Rickets and Severe Hypocalcemia in Infants Fed Homemade Alkaline Diet Formula - Three States, August 2020-February 2021 \\ 1127 QuickStats}

Continuing Education examination available at https://www.cdc.gov/mmwr/mmwr_continuingEducation.html

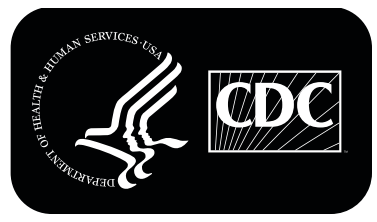

U.S. Department of Health and Human Services Centers for Disease Control and Prevention 
management regarding employee health and exposures, facility and equipment, food sources, and pest control. No further epidemiologic link was established from the interview with the restaurant, but continued cleanliness and maintenance citations occurred during 2014-2018. Additional Salmonella Mbandaka cases in 2017 prompted development of a detailed, outbreak-specific case questionnaire that included specific questions about restaurant A. As more cases were identified, an intensive investigation began in 2018. An outbreak case was defined as a case of confirmed Salmonella Mbandaka with one of two closely related PFGE patterns (TDRX01.0120 and TDRX01.0127), highly related WGS subtype identified by the MDHHS Bureau of Laboratories or CDC, or probable cases with clinically compatible illness and epidemiologic linkage to a confirmed case.

During September 2008-July 2019, a total of 35 primary cases (33 confirmed and two probable) and one confirmed secondary case were identified. Patients with confirmed cases ranged in age from $1.5-90$ years $($ mean $=57$ years; median $=64$ years), and $26(72 \%)$ patients were female. Several patients reported a history of chronic gastrointestinal issues that made determination of onset date difficult. Twenty-four (67\%) patients reported vomiting or diarrhea, and $12(33 \%)$ reported urinary tract infection. Six (17\%) patients were hospitalized. Approximately $40 \%$ of patients had underlying medical conditions such as diabetes or cancer. Among 19 patients with a restaurant dining history, 17 reported eating at restaurant $\mathrm{A}$.
Patients were routinely interviewed at the time of local health department referral and reinterviewed, often weeks later, when Mbandaka serotype was reported. Thirteen patients, retrospectively identified from early in the outbreak period, were not candidates for reinterview because their onsets preceded identifying them as part of the outbreak by $>1$ month.

After implementation of the outbreak-specific questionnaire in 2017, nine patients with onset during August 2017July 2019 reported having eaten at restaurant A (Figure). To determine whether restaurant $A$ was mentioned in the food histories of other reported foodborne illnesses, Michigan public health officials reviewed restaurant A patronage and food histories of 1,166 persons with previously reported salmonellosis, campylobacteriosis, and shigellosis cases in southwest Michigan for restaurant dining history. The only patients who reported eating at restaurant $A$ were those associated with this outbreak; no other patients mentioned the restaurant.

\section{Environmental and Laboratory Investigation}

As part of the 2018 investigation, restaurant A employee stool specimens and environmental samples were collected in parallel and analyzed for Salmonella. None of the 100 employees reported symptoms at the time of sample collection or in the weeks preceding collection. MDHHS Bureau of Laboratories identified five isolates from four of 100 asymptomatic employees' stool specimens that shared the outbreak subtype. Stool cultures were collected approximately every 30 days from

The MMWR series of publications is published by the Center for Surveillance, Epidemiology, and Laboratory Services, Centers for Disease Control and Prevention (CDC), U.S. Department of Health and Human Services, Atlanta, GA 30329-4027.

Suggested citation: [Author names; first three, then et al., if more than six.] [Report title]. MMWR Morb Mortal Wkly Rep 2021;70:[inclusive page numbers].

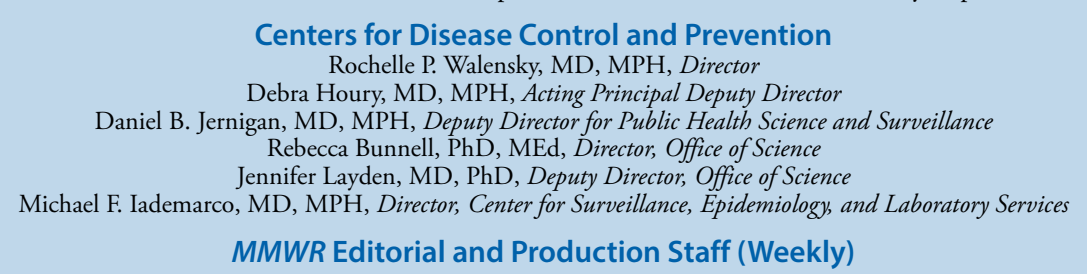

MMWR Editorial and Production Staff (Weekly)

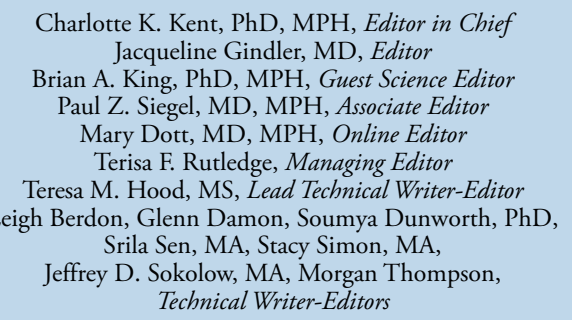

Martha F. Boyd, Lead Visual Information Specialist Alexander J. Gottardy, Maureen A. Leahy, Julia C. Martinroe, Stephen R. Spriggs, Brian Taitt, Tong Yang, Visual Information Specialists

Quang M. Doan, MBA, Phyllis H. King, Terraye M. Starr, Moua Yang, Ian Branam, MA, Ginger Redmon, MA, Co-Acting Lead Health Communication Specialists Shelton Bartley, MPH, Lowery Johnson, Amanda Ray, Jacqueline N. Sanchez, MS, Health Communication Specialists Will Yang, MA,

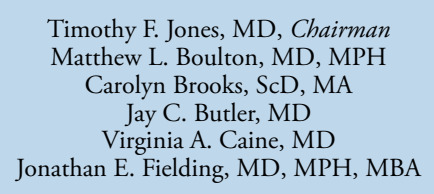

Patrick L. Remington, MD, MPH Carlos Roig, MS, MA William Schaffner, MD Nathaniel Smith, MD, MPH Morgan Bobb Swanson, BS Abbigail Tumpey, MPH 
FIGURE. Cases of Salmonella Mbandaka outbreak subtype $(\mathrm{N}=35)$, by month and year of illness onset* and restaurant A exposure — Michigan, September 2008-July $2019^{\dagger}$

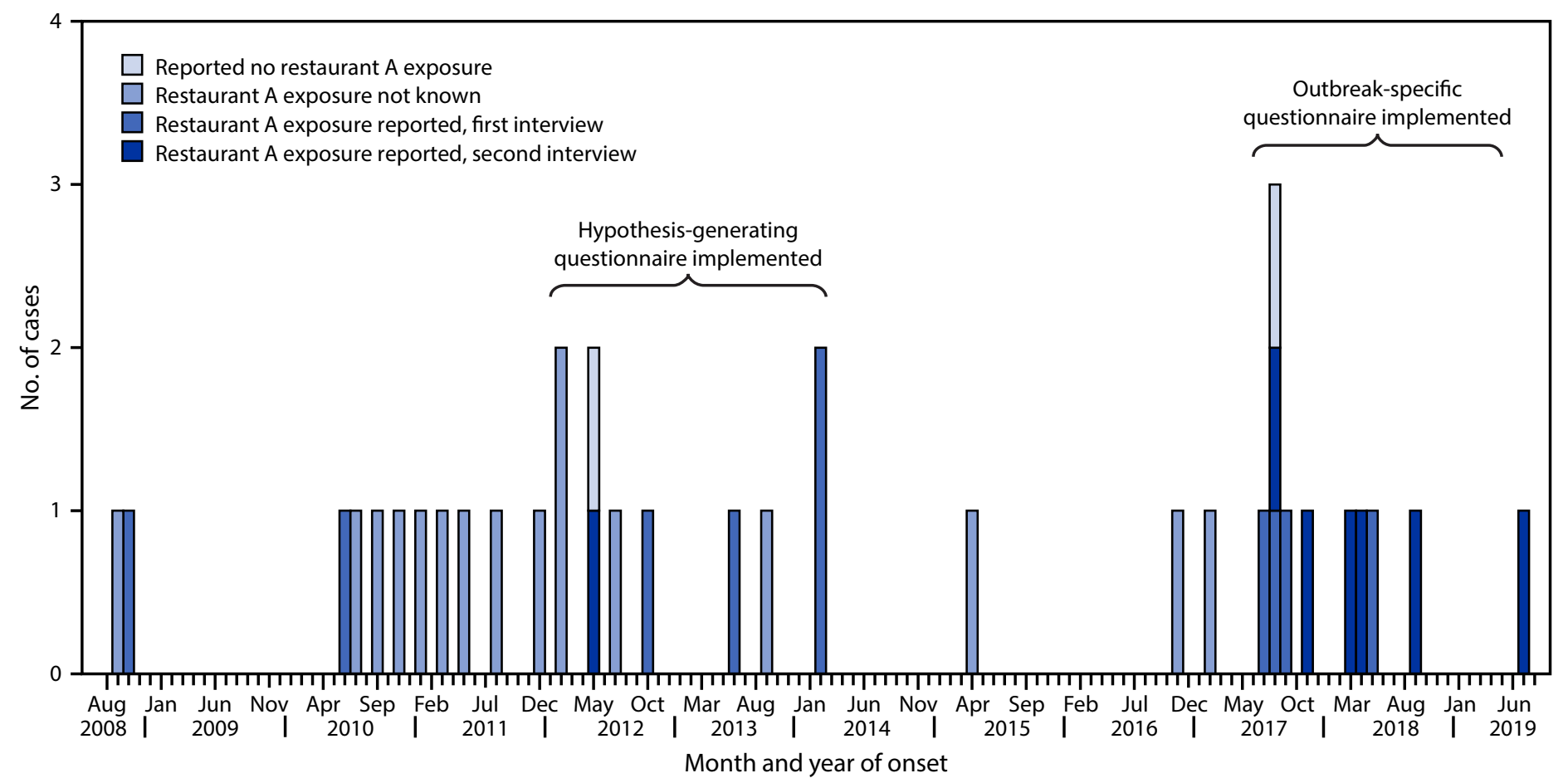

* Onset date was missing for five patients; for these cases, the date of referral to the health department was used.

† Pulsed-field gel electrophoresis was performed only on the top 20 Salmonella serotypes submitted to the Michigan Department of Health and Human Services Bureau of Laboratories from approximately 2009 to early 2010; the Salmonella Mbandaka serotype was rare and not a top 20 serotype.

asymptomatic employees with stool specimens that tested positive until a negative test result was received. A repeat stool culture was taken at least 48 hours after the first negative result. Asymptomatic employees who received positive Salmonella Mbandaka test results were required to have two negative stool cultures before returning to work. Because antibiotics can increase the likelihood of prolonged Salmonella shedding in stool, treatment was not recommended (1). None of the restaurant employees received antibiotics to treat asymptomatic Salmonella Mbandaka infection. The duration of Salmonella shedding among the four asymptomatic restaurant employees with positive cultures varied (range $=31-123$ days).

A team of local and state public health officials collected 80 environmental samples from food and nonfood contact surfaces to be tested for Salmonella. Salmonella was isolated from 39 (49\%) environmental samples. Positive samples shared the same PFGE and WGS results as the outbreak subtype (Table). Positive samples were collected throughout the restaurant kitchen, including cooking, preparation, dishwashing, storage, and employee restroom areas. Following the identification of a new case in September 2018, a second round of environmental Salmonella sampling was conducted. In the second round, Salmonella was isolated from 11 of 81 samples (14\%) and shared the outbreak subtype. Positive environmental sites were generally similar, but not identical, in the two rounds. Environmental, asymptomatic employee, and symptomatic patient isolates identified by core genome multilocus sequence typing (cgMLST) revealed three clades (A, B, and C) (Table). Isolates within each clade were highly related, differing by $\leq 5$ alleles. The clades were also considered highly related to each other, differing by $\leq 15$ alleles. All environmental and employee isolates were in clade $A$; symptomatic patient isolates were identified in all three clades.

\section{Public Health Response}

In addition to routine inspections and administrative hearings in 2012 and 2014, iterative facility environmental assessments and administrative hearings during 2017-2018 addressed cleanliness, lack of active managerial control, and other foodborne illness risk factors cited at restaurant A. In addition to employee stool screening for Salmonella and exclusion of asymptomatic employees with Salmonella stool positive results, employees were also required to submit stool specimens for Salmonella testing if new onset of gastrointestinal symptoms occurred during the 2018 public health response. Seven employees reported symptoms, but all had 
Morbidity and Mortality Weekly Report

TABLE. Characteristics of Salmonella Mbandaka outbreak subtype isolates from symptomatic patients, asymptomatic restaurant A employees, and restaurant A environmental surfaces - Michigan, August 2008-June 2018

\begin{tabular}{|c|c|c|c|c|c|}
\hline Source of isolate & $\begin{array}{l}\text { No. of samples } \\
\text { collected }\end{array}$ & $\begin{array}{l}\text { No. }(\%) \text { of isolates } \\
\text { identified by PFGE }\end{array}$ & $\begin{array}{l}\text { No. (\%) of isolates } \\
\text { identified by WGS }\end{array}$ & $\begin{array}{l}\text { Isolation date or } \\
\text { date range }\end{array}$ & Clade by cgMLST \\
\hline \multirow[t]{3}{*}{ Symptomatic patient } & 36 & $36(100)$ & $30(83)$ & 2008-2012 & B \\
\hline & & & & 2012-2014 & $\mathrm{C}$ \\
\hline & & & & $2015-2018$ & $A$ \\
\hline Asymptomatic employee* & 100 & $5(5)$ & $5(5)$ & Jun 2018 & $A$ \\
\hline Environment (restaurant) & 80 & $39(49)$ & $26(33)$ & Jun 2018 & $A$ \\
\hline Environment (restaurant) & 81 & $11(14)$ & $10(12)$ & Nov 2018 & $A$ \\
\hline
\end{tabular}

Abbreviations: cgMLST = core genome multilocus sequence typing; PFGE = pulsed-field gel electrophoresis; WGS = whole genome sequencing

* Five isolates were analyzed from four asymptomatic employees.

negative Salmonella test results. Before the initial environmental Salmonella sampling event in spring 2018, the facility temporarily closed for renovations of the kitchen, flooring, walls, and major equipment. The restaurant was required to clean all facility and food contact surfaces to norovirus cleaning standards* and underwent follow-up environmental Salmonella sampling. In fall 2018, the facility temporarily closed for additional floor and equipment renovations with the intent of eradicating Salmonella from the facility. The facility again had a full norovirus standard cleaning performed before reopening. Despite kitchen renovation and environmental hygiene interventions, Salmonella Mbandaka continued to be detected in restaurant $\mathrm{A}$. Therefore, the restaurant voluntarily and permanently closed in late 2018 , and food, dishes, storage, soft goods, chairs, and tables were destroyed. The metal food production equipment was extensively cleaned, quarantined, and resampled for Salmonella before being redeployed. The building was deemed ineligible for food production or storage relicensure. One case of the outbreak subtype was isolated in urine 8 months after closure of the restaurant; the patient reported chronic, intermittent diarrhea after eating at restaurant A 3 weeks before it closed.

\section{Discussion}

Multiple challenges contributed to delayed source identification. Food histories were incomplete in the early cases. Initial questionnaires were inflexible and focused more on food items than on food establishments. Early cases were not initially identified as a cluster given the sporadic incidence and were hypothesized as a rare or regional PFGE pattern. Further, index of suspicion for a protracted common source early in the outbreak was low given the more typical experience of point source Salmonella outbreaks. Finally, restaurant management was doubtful and required intensive engagement. Both the environmental and clinical testing results were thus essential for continued mitigation efforts.

\footnotetext{
${ }^{*}$ https://www.cdc.gov/norovirus/about/prevention.html
}

Salmonellosis outbreaks in the food industry often occur through a point source when undercooked or contaminated food products infect consumers until distribution of the foodborne vehicle ceases $(2,3)$. In this outbreak, a complex association between the environment and employees of a single restaurant in southwest Michigan demonstrated a protracted and intermittent common source outbreak of Salmonella Mbandaka. A study of 23 restaurant-associated salmonellosis outbreaks found that restaurants with Salmonella-positive environmental samples had a higher proportion of Salmonellapositive food workers and longer outbreak durations than did restaurants with negative environmental samples (4). The nearly 11-year duration of this outbreak attests to the potential recalcitrance of Salmonella in restaurant environments, the importance of hygienic restaurant policies and practices, and the challenge in source identification when cases occur intermittently and without a clear foodborne vehicle. As WGS is more broadly implemented as a routine subtyping method for Salmonella and other bacterial enteric pathogens, increased discriminatory power might facilitate the identification of more protracted, common-source outbreaks (5). Whereas initially small numbers of cases might present a challenge to definitively implicating a common source, gathering as much high quality exposure data as possible, including repeated interviewing of patients with cases that are clustered in time using closed-ended questions about exposures of interest, can aid an investigation. In addition, conducting environmental assessments, environmental sampling, and employee testing for Salmonella are best practices that should be considered early in an investigation, particularly when a single foodborne vehicle is not apparent.

Fifteen (42\%) of the 36 patients had the outbreak subtype isolated in urine; $12(33 \%)$ patients had urinary symptoms without reporting diarrhea or vomiting. These findings are consistent with the observation that a higher proportion of Salmonella serogroup C1 (including Mbandaka) than of other Salmonella serogroups is isolated from urine $(6,7)$. Although chronic carriage of Salmonella Typhi after acute infection is widely recognized, asymptomatic carriage of nontyphoidal 


\section{Summary}

What is already known about this topic?

Restaurant outbreaks of Salmonella with Salmonella-positive environmental samples might result in a higher proportion of Salmonella-positive food workers and longer outbreaks.

What is added by this report?

A protracted restaurant-associated outbreak of Salmonella Mbandaka in Michigan was identified through recursive case interviewing, asymptomatic employee testing, and environmental sampling. Multiple efforts to eradicate the organism failed, and the restaurant was permanently closed in 2018.

What are the implications for public health practice?

Coupling asymptomatic food worker testing and environmental sampling for Salmonella with whole genome sequencing of case isolates in suspected, protracted restaurant outbreaks of Salmonella enhances source identification. Exclusion or restriction of asymptomatic food workers with nontyphoidal Salmonella should be considered part of restaurant outbreak mitigation.

Salmonella is less well characterized but has been reported in restaurant food and hotel workers as well as in healthy adults and children $(5,8,9)$.

For most of the time when the reported outbreak investigation was conducted, the restaurant was regulated under a modified version of the 2009 Food and Drug Administration (FDA) Food Code, the latest FDA Food Code that Michigan had adopted. The 2009 FDA Food Code did not include asymptomatic nontyphoidal Salmonella infections among the five specific foodborne pathogens ${ }^{\dagger}$ for which exclusion and restriction requirements are delineated. Therefore, the 2017 FDA Food Code was used for guidance because it includes asymptomatic nontyphoidal Salmonella infection as a food worker condition of restriction (10). Further adoption of the 2017 FDA Food Code will aid public health professionals in disrupting nontyphoidal Salmonella transmission in restaurant settings, particularly as more protracted outbreaks are identified.

\footnotetext{
${ }_{\dagger}^{\dagger}$ Norovirus, Salmonella Typhi, Escherichia coli O157:H7 or Enterohemorrhagic or Shiga toxin-producing E. coli, Shigella spp., and hepatitis A virus.
}

\section{Acknowledgments}

Penny Born, Ashley Huver, Vern Johnson, Nicole Wilson, Kalamazoo County Health and Community Services Department; Leslie Dybas, Tiffany Henderson, Karen Pietrzen, Jason Wholehan, Michigan Department of Health and Human Services; Lisa Hainstock, William Hull, Michigan Department of Agriculture and Rural Development; Eija Trees, PulseNet Next Generation Subtyping Methods Unit, CDC; PulseNet Outbreak Detection and Surveillance Unit, CDC.
Corresponding author: William Nettleton, wdnett@kalcounty.com, 269-373-5261.

${ }^{1}$ Kalamazoo County Health and Community Services Department, Kalamazoo,
Michigan; ${ }^{2}$ Department of Family and Community Medicine, Western
Michigan University Homer Stryker M.D. School of Medicine, Kalamazoo,
Michigan; ${ }^{3}$ Michigan Department of Health and Human Services.

All authors have completed and submitted the International Committee of Medical Journal Editors form for disclosure of potential conflicts of interest. No potential conflicts of interest were disclosed.

\section{References}

1. Onwuezobe IA, Oshun PO, Odigwe CC. Antimicrobials for treating symptomatic non-typhoidal Salmonella infection. Cochrane Database Syst Rev 2012;11:CD001167. PMID:23152205 https://doi. org/10.1002/14651858.CD001167.pub2

2. Dewey-Mattia D, Manikonda K, Hall AJ, Wise ME, Crowe SJ. Surveillance for foodborne disease outbreaks-United States, 20092015. MMWR Surveill Summ 2018;67(No. SS-10). PMID:30048426 https://doi.org/10.15585/mmwr.ss6710a1

3. Angelo KM, Nisler AL, Hall AJ, Brown LG, Gould LH. Epidemiology of restaurant-associated foodborne disease outbreaks, United States, 1998-2013. Epidemiol Infect 2017;145:523-34. PMID:27751201 https://doi.org/10.1017/S0950268816002314

4. Medus C, Smith KE, Bender JB, Besser JM, Hedberg CW. Salmonella outbreaks in restaurants in Minnesota, 1995 through 2003: evaluation of the role of infected foodworkers. J Food Prot 2006;69:1870-8. PMID:16924912 https://doi.org/10.4315/0362-028X-69.8.1870

5. Mair-Jenkins J, Borges-Stewart R, Harbour C, et al. Investigation using whole genome sequencing of a prolonged restaurant outbreak of Salmonella Typhimurium linked to the building drainage system, England, February 2015 to March 2016. Euro Surveill 2017;22:17-37. PMID:29233257 https://doi.org/10.2807/1560-7917.ES.2017.22.49.17-00037

6. CDC. An atlas of Salmonella in the United States, 1968-2011. Atlanta, GA: US Department of Health and Human Services, CDC; 2013. https://www.cdc.gov/salmonella/pdf/salmonella-atlas-508c.pdf

7. Abbott SL, Portoni BA, Janda JM. Urinary tract infections associated with nontyphoidal Salmonella serogroups. J Clin Microbiol 1999;37:4177-8. PMID:10565958 https://doi.org/10.1128/JCM.37.12.4177-4178.1999

8. Sirinavin S, Pokawattana L, Bangtrakulnondh A. Duration of nontyphoidal Salmonella carriage in asymptomatic adults. Clin Infect Dis 2004;38:1644-5. PMID:15156460 https://doi.org/10.1086/421027

9. Gunn JS, Marshall JM, Baker S, Dongol S, Charles RC, Ryan ET. Salmonella chronic carriage: epidemiology, diagnosis, and gallbladder persistence. Trends Microbiol 2014;22:648-55. PMID:25065707 https://doi.org/10.1016/j.tim.2014.06.007

10. Food and Drug Administration. Food code, 2017. Silver Spring, MD: US Department of Health and Human Services, Food and Drug Administration; 2019. https://www.fda.gov/food/fda-food-code/ food-code-2017 